\title{
Aclaramiento Dental con Enjuagues de Libre Venta que Contienen Peróxido de Hidrógeno
}

\author{
Toothbleaching with over the Counter Mouthwashes Containing Hydrogen Peroxide
}

\author{
Rosa Josefina Roncal Espinozaํㅛ \&idia Yileng Tay Chu Jon²
}

RONCAL, R. J. \& TAY, L. Y. Aclaramiento dental con enjuagues de libre venta que contienen peróxido de hidrógeno. Int. J. Odontostomat., 12(2):121-124, 2018.

RESUMEN: El objetivo de este estudio in vitro fue evaluar la eficacia en el aclaramiento dental de tres enjuagues orales que contienen peróxido de hidrógeno en diferentes períodos de inmersión en comparación con el peróxido de carbamida al $10 \%$. Cuarenta muestras de premolares humanos se dividieron aleatoriamente en cuatro grupos según el agente al que se expuso: G1: Colgate Plax®Whitening, G2: Listerine ${ }^{\circledR}$ Whitening Extreme y G3: Oral $B^{\circledR} 3 D$ White ${ }^{\text {TM }}$ se sumergieron dos minutos al día durante 28 días y G4: Peróxido de carbamida al $10 \%$, ocho horas al día durante 14 días. La medición de color se realizó con un espectrofotómetro Vita Easyshade usando la escala CIELab inicialmente, a los 14,28 y 35 días de evaluación. Las comparaciones entre los grupos se realizaron utilizando las pruebas Kruskal-Wallis y U Mann-Whitney, mientras que entre los tiempos las pruebas Friedman y Signo-Rango de Wilcoxon. Los resultados revelaron que a los 14 días, los cuatro grupos mostraron cambios de color, pero no se evidenció mayor eficacia de alguno sobre el otro $(p>0,05)$. Sin embargo, a los 28 y 35 días se observó una clara eficacia del peróxido de carbamida sobre los enjuagues ( $p<0,05)$. Los enjuagatorios orales aclaran los dientes a partir de los 28 días, con los protocolos indicados; sin embargo no llegan a tener resultados similares a un aclaramiento profesional con gel de peróxido de carbamida al $10 \%$.

PALABRAS CLAVE: blanqueamiento dental, blanqueador de dientes, peróxido de hidrógeno, enjuagues orales, espectrofotometría.

\section{INTRODUCCIÓN}

El cambio de color en los dientes tiene lugar durante el tiempo y puede tener orígenes extrínsecos, cuando existe una aglutinación de alimentos pigmentados en la superficie dental e intrínsecos relacionados con la presencia de sustancias cromóforas al interior del tejido dentinario o adamantino (Barry et al., 2017).

El aclaramiento dental constituye una solución para este cambio de coloración, existiendo para ello un gran número de productos a base de peróxido de carbamida o peróxido de hidrógeno, que se descompone en oxígeno y penetra en las porosidades del tejido dental descomponiendo moléculas cromóforas que dan color al diente (Jaime et al., 2014).

Este tratamiento, actualmente es ampliamente utilizado, no sólo de modo profesional, sino también como productos de libre venta (OTC), con niveles más bajos de agente blanqueador y auto aplicables por el paciente, éstos pueden ser: dentífricos, colutorios, tiras de aclaramiento, etc. (Jaime et al.; Majeed et al., 2015).

Los enjuagues orales actúan controlando químicamente biofilms cariogénicos y presentan propiedades remineralizantes. Hoy en día, debido a la creciente preocupación de los pacientes por la estética dental el número de estos productos conteniendo peróxido de hidrógeno ha aumentado significativamente (Karadas \& Hatipoglu, 2105).

Por ello, este estudio evalúa la eficacia in vitro de tres enjuagues orales que contienen peróxido de hidrógeno: Colgate Plax®Whitening, Listerine $₫ W h i t e n i n g$ Extreme y Oral-B $\AA 3 D$ White ${ }^{\mathrm{TM}}$, en comparación con un gel blanqueador de uso profesional odontológico a

\footnotetext{
${ }^{1}$ Docente de la Facultad de Odontología de la Universidad Católica Santo Toribio de Mogrovejo, Chiclayo, Perú.

2 Docente de la Facultad de Estomatología Universidad Peruana Cayetano Heredia, Lima, Perú.
} 
base peróxido de carbamida (Opalescence - Jeringa con $3 \mathrm{~g}$ al 10\%), en diferentes períodos de inmersión.

\section{MATERIAL Y MÉTODO}

Este estudio incluyó premolares humanos extraídos por motivos ortodónticos, excluyendo dientes con caries, desgastados, restaurados o pigmentados. Los dientes seleccionados, se seccionaron a nivel de la unión cemento adamantina para obtener las coronas como muestras. Cada muestra fue montada individualmente en acrílico exponiendo la superficie del esmalte, siendo sumergidas durante tres días en una mezcla de té negro (Mac Colin's MR), para homogenizar el color inicial de los dientes.

Las muestras pigmentadas fueron asignadas aleatoriamente en cuatro grupos $(n=10)$ según el enjuagatorio al que se expuso: G1: Colgate Plax® Whitening (peróxido de hidrógeno al 1,5 \%), G2: Listerine ${ }^{\circledR}$ Whitening Extreme (peróxido de hidrógeno al 2,5\%), G3: Oral- B® 3D White ${ }^{\mathrm{TM}}$ (peróxido de hidrógeno al $1,5 \%$ ) y G4: gel blanqueador Opalescence $10 \%$ (peróxido de carbamida al $10 \%$ - control positivo). Los grupos $\mathrm{G} 1, \mathrm{G} 2$ y G3 se sumergieron a $37^{\circ} \mathrm{C}$ en $40 \mathrm{ml}$ de enjuague bucal durante dos minutos al día por 28 días. Además de los 28 días, las muestras fueron sumergidas 7 días más en agua destilada para evaluar la estabilidad del color. En el grupo control se colocó una capa de gel blanqueador (Opalescence $10 \%$ ), extendiéndola sobre la superficie del esmalte 8 horas al día durante 14 días.

El investigador, previamente capacitado por un examinador entrenado, realizó las mediciones de color de cada muestra sobre un fondo blanco con luz de día, utilizando un espectrofotómetro digital (VITA Easyshade 4.0) que se calibró según las instrucciones del fabricante. Se registraron: $L^{*}, C^{*}, h^{*}$ y $a{ }^{*} b$ * que representan valores de coordenadas creados por la Commission Internationale de l'Éclairage. El valor $L$ * representa el grado de luminosidad y varía desde el negro (0) a blanco (100). El valor $h^{*}$ representa el matiz o tono. El valor $C^{*}$ indica el croma. $a *{ }^{*}$ * muestran el grado de rojo (+ $\left.a^{*}\right)$ - verde $(-a *)$, amarillo $\left(+b^{*}+\right)$ - azul $\left(-b^{*}\right)$. La toma de color se realizó en cuatro tiempos: después de la tinción con té (tiempo 0); a los 14 días de exposición (tiempo 1), 28 días de exposición (tiempo 2), 7 días después de terminada la exposición (tiempo 3 ) para evaluar la estabilidad del color, es decir, a los 35 días de empezado el estudio. El $\Delta \mathrm{E}$ se calculó mediante la fórmula: $\Delta \mathrm{E}=\left[\left(\Delta \mathrm{L}^{*}\right) 2+\left(\Delta \mathrm{a}{ }^{*}\right) 2+\left(\Delta \mathrm{b}^{*}\right) 2\right] 1 / 2$, que expresa la diferencia cromática total del diente (Karadas \& Hatipoglu).

Las comparaciones entre los grupos se realizaron utilizando las pruebas Kruskal-Wallis y U MannWhitney, mientras que entre los tiempos las pruebas Friedman y Signo-Rango de Wilcoxon, con un nivel de significancia de $95 \%$, usando el programa SPSS Statistics versión 22.0 (IBM, Armonk, NY, USA).

Tabla I. Media y desviación estándar de las dimensiones de color de las muestras en diferentes períodos de inmersión usando tres enjuagues orales que contienen peróxido de hidrógeno en comparación con el efecto blanqueador del peróxido de carbamida al $10 \%$.

\begin{tabular}{|c|c|c|c|c|c|c|}
\hline Tiempo & Enjuagatorios & $\mathrm{N}$ & $\begin{array}{l}\Delta \mathrm{E} \\
\mathrm{X}(\mathrm{DE})\end{array}$ & $\begin{array}{l}\Delta \mathrm{L} \\
\mathrm{X}(\mathrm{DE})\end{array}$ & $\begin{array}{l}\Delta \mathrm{C} \\
\mathrm{X}(\mathrm{DE})\end{array}$ & $\begin{array}{l}\Delta \mathrm{H} \\
\mathrm{X}(\mathrm{DE})\end{array}$ \\
\hline \multirow{5}{*}{14 días } & G1 & 10 & $3.77(2.54)$ & $5.10^{1,4}(14.95)$ & $4.77(8.76)$ & $2.09^{1,4}(14.88)$ \\
\hline & G2 & 10 & $2.44(3.38)$ & $3.96^{3,4}(15.71)$ & $6.63(2.92)$ & $0.33^{3}(2.08)$ \\
\hline & G3 & 10 & $3.28(2.30)$ & $0.37^{1,3}(5.30)$ & $6.36(2.53)$ & $-1.09^{2,3}(1.81)$ \\
\hline & G4 & 10 & $5.13(7.71)$ & $-2.45(12.41)$ & 9.04 (5.96) & $-2.134(2.55)$ \\
\hline & $p^{*}$ & & 0.256 & $<0.001$ & 0.128 & 0.004 \\
\hline \multirow{4}{*}{28 días } & G1 & 10 & $3.37^{1,4}(4.35)$ & $-0.91(3.15)$ & $5.36^{1,4}(2.67)$ & $-1.874(1.49)$ \\
\hline & G2 & 10 & $3.45^{3,4}(4.89)$ & $1.41(4.65)$ & $6.24^{3,4}(5.08)$ & $-1.454(2.71)$ \\
\hline & G3 & 10 & $2.29^{1,3}(4.48)$ & $-1.53(5.51)$ & $3.03^{1,3}(6.09)$ & $-1.96^{1,3}(2.16)$ \\
\hline & G4 & 10 & $7.55(5.35)$ & $-0.62(5.23)$ & $11.87(5.45)$ & $-3.47(2.10)$ \\
\hline \multirow{6}{*}{35 días } & $p^{*}$ & & $<0.001$ & 0.281 & $<0.001$ & 0.001 \\
\hline & $\mathrm{G} 1$ & 10 & $4.60^{1,4}(3.63)$ & $-0.83^{1,2,4}(2.48)$ & $6.45^{4}(2.61)$ & $-2.714(1.29)$ \\
\hline & G2 & 10 & $2.73^{3,4}(4.48)$ & $1.12^{3}(4.70)$ & $2.37(4.57)$ & $-0.29(2.57)$ \\
\hline & G3 & 10 & $3.03^{1,3}(4.74)$ & $-2.21^{2,3}(5.18)$ & $5.56^{3}(4.89)$ & $-2.483(2.19)$ \\
\hline & G4 & 10 & $9.89(5.30)$ & $-1.77^{3,4}(5.05)$ & $15.50(4.51)$ & $-4.18(1.97)$ \\
\hline & $p^{*}$ & & $<0.001$ & 0.009 & $<0.001$ & $<0.001$ \\
\hline
\end{tabular}

*Kruskal Wallis; DE, desviación estándar. Los superíndices indican los números con las cuales no difieren estadísticamente (U de Mann-Whitney $p<0,05$ ). 


\section{RESULTADOS}

Los valores de *a y *b mostraron diferencias significativas en el grupo control respecto a los enjuagatorios. Los grupos G1, G2 y G3 mostraron variaciones irregulares en el tiempo.

Los resultados mostraron diferencias significativas a los 14 días, con mayores puntuaciones de $\Delta \mathrm{L}$ para los enjuagatorios respecto al control y mayores puntuaciones de $\Delta \mathrm{H}$ para Listerine y Colgate respecto al control $(p<0,05)$. A los 28 días todos los colutorios mostraron menores puntuaciones de $\Delta \mathrm{E}$ y $\Delta \mathrm{C}$ que el control, pero mayores puntuaciones de $\Delta \mathrm{H}(p<0,05)$, lo mismo ocurrió a los 35 días $(p<0,05)$ donde además sólo Listerine mostró mayor puntuación de $\Delta \mathrm{L}$ que el control (Tabla I).

\section{DISCUSIÓN}

Los tres enjuagues orales evaluados aclaran los dientes, sin embargo, resultan en un menor aclaramiento en comparación con el gel de peróxido de carbamida al $10 \%$. Estos resultados coinciden con los de Jaime et al. quienes concluyen que hubo un aumento significativo de la luminosidad en las muestras expuestas al gel de peróxido de carbamida (PC), así como una mejor eficacia de aclaramiento frente al enjuague oral aclarador. Además, Luque-Martínez et al. (2016) en un meta análisis compararon el cambio de color producido por el PC, frente a los productos a base de peróxido de hidrógeno $(\mathrm{PH})$, evidenciando que el PC tiene una mejor eficacia en el aclaramiento frente a los productos a base de $\mathrm{PH}$, probablemente debido a que los enjuagues orales contienen una concentración menor de HP que va entre 1 a 2,5.

Potgieter et al. (2011) evaluaron la eficacia de aclaramiento de tres enjuagues orales: Colgate Plax Whitening Blancheur, White Glo 2 en 1 y Plus White durante 21 días por un tiempo de dos minutos al día, dando como resultado que ninguno de los tres enjuagues orales mostraron efectos significativos en el aclaramiento, esto puede deberse a que los enjuagues mencionados no contienen peróxido de hidrógeno, o no precisan su concentración, usando como agente blanqueador abrasivos u otros productos.

En general, los resultados mostraron que el período de inmersión y los enjuagues orales tienen in- fluencia en los cambios de color. Los primeros 14 días los cuatro grupos aclararon pero no se evidenció mayor eficacia de alguno de estos sobre el otro. Sin embargo a los 28 días y 35 días se observa una clara eficacia del peróxido de carbamida sobre los enjuagatorios.

El tiempo de tratamiento influye en los resultados finales; Colgate Plax ${ }^{\circledR}$ Whitening a los 35 días presentó un efecto aclarador mayor que los demás enjuagatorios orales, sin ser éste más eficaz que el control. Esto coindice con el estudio de Demarco et al. (2009) en el cual se concluye que los geles y enjuagues producen un efecto blanqueador que no parece ser clínicamente significativo. No obstante, Torres et al. (2013) mostraron que el cambio de color conseguido con enjuagues orales blanqueadores durante 12 semanas fue similar al resultado obtenido con PC al $10 \%$ utilizado durante 14 días.

Respecto a la luminosidad, todos los grupos presentaron variación, la misma que fue más evidente, con puntuaciones negativas, que quieren decir valores más cercanos al blanco en el grupo control y Oral $B \otimes 3 D$ White ${ }^{\mathrm{TM}}$, a los 35 días, y resultando con efectos menos luminosos el Listerine ${ }$ Whitening Extreme. Estos resultados no coinciden con el estudio de Lima et al. (2012) quienes evaluaron el efecto del aclaramiento con dos enjuagues bucales: Listerine $\AA$ Whitening y Colgate Plax ${ }^{\circledR}$ Whitening en un período de 45 días, dos veces al día durante un minuto; mostrando que ambos productos presentaron un efecto blanqueador, sin embargo Colgate Plax® Whitening mostró una reducción en la luminosidad de las muestras, debido a que los enjuagues bucales presentan un $\mathrm{pH}$ de $5,6 \mathrm{y}$ la exposición continua de los dientes a productos ácidos puede originar incorporación de pigmentos ocasionando su oscurecimiento.

En cuanto a la tonalidad cromática varía significativamente entre los grupos presentando una coloración más amarillenta el grupo de ColgatePlax ${ }^{\circledR}$ Whitening a los 14 días, seguido por Listerine Whitening Extreme. Esto podría haber ocurrido por la concentración de $\mathrm{PH}$ que es mayor en el Listerine Whitening Extreme.

Cabe señalar, que el aclaramiento ocurre al aumentar la luminosidad, disminuir la concentración de amarillo (disminución de *b), y, reducir la concentración de rojo (disminución de *a). Este estudio resultó en la disminución del valor de *a y *b en el grupo control, corroborando la eficacia del grupo control en el 
aclaramiento dental. Así mismo, se evidenció la inestabilidad en el tiempo para el grupo Listerine Whitening Extreme, pues a pesar de presentar mayor concentración de HP que el resto de enjuagues bucales, presenta bajo $\mathrm{pH}$ lo que puede contribuir en su inestabilidad de color.

Pese a que se requiere un seguimiento preferentemente en estudios in vivo, esta investigación es importante ya que revela resultados favorables de un tratamiento respaldado científicamente bajo asesoría profesional (gel blanqueador Opalescence $10 \%$ ), respecto a los enjuagues orales, productos de venta libre, que tienden a aclarar ligeramente los dientes, pero que su eficacia es menor y cuyos efectos colaterales se desconocen.

RONCAL, R. J. \& TAY, L. Y. Toothbleaching with free-sale mouthwashes containing hydrogen peroxide. Int. J. Odontostomat., 12(2):121-124, 2018.

ABSTRACT : The objective of this in vitro study was to evaluate the efficacy in dental toothbleaching of three mouthwashes containing hydrogen peroxide in different periods of immersion compared to $10 \%$ carbamide peroxide. Forty samples of human premolars were randomly divided into four groups according to the agent to which they were exposed: G1: Colgate Plax®Whitening, G2: Listerine ${ }^{\circledR} W h$ itening Extreme and G3: Oral $B \otimes 3 D$ White ${ }^{T M}$ were immersed two minutes a day during 28 days and G4: $10 \%$ carbamide peroxide, eight hours a day for 14 days. The color measurement was performed with a Vita Easyshade spectrophotometer using the CIELab scale initially, at 14, 28 and 35 days of evaluation. The comparisons between the groups were made using the Kruskal-Wallis and $U$ Mann-Whitney tests. While between the times the tests Friedman and Sign-Range of Wilcoxon. The results revealed that at 14 days, the four groups showed color changes, but no greater effectiveness was shown of one over the other $(p>0,05)$. However, at 28 and 35 days a clear efficacy of carbamide peroxide was observed on the rinses $(p<0,05)$. Mouthwashes clear the teeth after 28 days, with the indicated protocols; however, they do not reach similar results to a professional clarification with $10 \%$ carbamide peroxide gel.

KEY WORDS: toothbleaching, tooth-bleaching agent, hydrogen peroxide, mouthwashes, spectrophotometry.

\section{REFERENCIAS BIBLIOGRÁFICAS}

Barry, T. N.; Bailey, C. W.; Ashcraft-Olmscheid, D. \& Vandewalle, K. $\mathrm{S}$. Effect of a new bleaching gel on tooth whitening. Oper. Dent., 42(5):559-66, 2017.

Demarco, F. F.; Meireles, S. S. \& Masotti, A. S. Over-the-counter whitening agents: a concise review. Braz. Oral Res., 23 Suppl. 1:64-70, 2009.
Jaime, I. M.; França, F. M.; Basting, R. T.; Turssi, C. P. \& Amaral, F. L. Efficacy of hydrogen-peroxide-based mouthwash in altering enamel color. Am. J. Dent., 27(1):47-50, 2014

Karadas, M. \& Hatipoglu, O. Efficacy of mouthwashes containing hydrogen peroxide on tooth whitening. Sci. World J., 2015:961403, 2015.

Lima, F. G.; Rotta, T. A.; Penso, S.; Meireles, S. S. \& Demarco, F. F. In vitro evaluation of the whitening effect of mouth rinses containing hydrogen peroxide. Braz. Oral Res., 26(3):269-74, 2012.

Luque-Martinez, I.; Reis, A.; Schroeder, M.; Muñoz, M. A.; Loguercio, A. D.; Masterson, D. \& Maia, L. C. Comparison of efficacy of tray-delivered carbamide and hydrogen peroxide for at-home bleaching: a systematic review and meta-analysis. Clin. Oral Investig., 20(7):1419-33, 2016.

Majeed, A.; Farooq, I.; Grobler, S. R. \& Moola, M. H. In vitro evaluation of variances between real and declared concentration of hydrogen peroxide in various tooth-whitening products. Acta Odontol. Scand., 73(5):387-90, 2015.

Potgieter, E. \& Grobler, S. R. Whitening efficacy of three over-thecounter oral rinses. S. A. D. J., 66(3):128-31, 2011.

Torres, C. R.; Perote, L. C.; Gutierrez, N. C.; Pucci, C. R. \& Borges, A. B. Efficacy of mouth rinses and toothpaste on tooth whitening. Oper. Dent., 38(1):57-62, 2013.

Dirección para correspondencia

Rosa Roncal Espinoza

Av San Josemaría Escriva de Balaguer 855

Universidad Católica Santo Toribio de Mogrovejo

Chiclayo

PERÚ

Email: rosijo312@hotmail.com

Recibido : 29-01-2018

Aceptado: 03-04-2018 